\title{
Understanding scale invariance in a minimal model of complex relaxation phenomena
}

\author{
P.I. Hurtado ${ }^{\dagger}$, J. Marro and P.L. Garrido \\ Institute Carlos I for Theoretical and Computational Physics, and \\ Departamento de Electromagnetismo y Física de la Materia, \\ Universidad de Granada, E-18071-Granada, España
}

November 19, 2018

\begin{abstract}
We report on the computer study of a lattice system that relaxes from a metastable state. Under appropriate nonequilibrium randomness, relaxation occurs by avalanches, i.e., the model evolution is discontinuous and displays many scales in a way that closely resembles the relaxation in a large number of complex systems in nature. Such apparent scale invariance simply results in the model from summing over many exponential relaxations, each with a scale which is determined by the curvature of the domain wall at which the avalanche originates. The claim that scale invariance in a nonequilibrium setting is to be associated with criticality is therefore not supported. Some hints that may help in checking this experimentally are discussed.

Key words: nonequilibrium, relaxation, metastable states, avalanches, scale invariance, $1 / f$ noise.
\end{abstract}

\section{Introduction}

Scaling behavior described by a simple power law is ubiquitous in nature. This is endowed a great theoretical interest on the assumption that it reveals prevalence of some underlying feature [1]-[7]. Power-law distributed events have no typical size, known as "scale invariance". The spectral density also reveals multiplicity of scales in many time series, i.e., the mean square fluctuation goes inversely with frequency, known as " $1 / f$ noise".

The concept of scale invariance originated in equilibrium statistical mechanics. This predicts dramatic extension of correlations near critical points; any microscopic spontaneous fluctuation then triggers events of any possible size with the same cause. Thermodynamic equilibrium is a special, pathological case, however, and deep understanding of why complex - out of equilibriumsystems are capable of events of any size, e.g., crashes in the stock market or disastrous earthquakes, is lacking.

Most of the cases already studied in detail, mainly those in the realm of physics, has now a model explanation. For example, the structured noise perceived in amplifiers besides a random spectrum [8] is explained based on the physics of the electron transport in a vacuum tube [9]; and the Barkhausen noise 10 is associated with the discontinuous motion of domain walls between pinned configurations in a disordered medium [1]. Specific models do not explain, however, the observed ubiquity and universality of the phenomenon. Among the interesting approaches that investigate this aspect, we mention SOC or selfadaptation into a critical condition [1]; the possibility that a system naturally lies on the edge between order and chaos [12; proximity to an standard critical point [13, 14, 15]; and the hypothesis that natural selection induces evolution towards a "highly structured state" [16. ${ }^{1}$ There is no full agreement yet on a globally coherent explanation, however. ${ }^{2}$

We present here a new effort towards better understanding this problem. We analyzed in detail a minimal model of relaxation phenomena which

\footnotetext{
${ }^{1}$ More specific mechanisms have also been proposed, including those in Refs. 17 - 25].

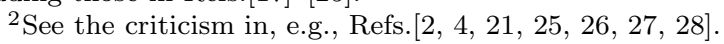


shows not only many scales but also some of the basic processes that typically characterize the natural phenomena of interest. In particular, under appropriate conditions, the model evolution proceeds via avalanches, each following a time plateau, and this occurs in a way that closely resembles the discontinuous variations with time of certain signals. For example, a similar relaxation process has been described concerning the current through resistors [21, the magnetization while a varying field induces domain rearrangements 29, 30, 11, the energy released in earthquakes [2, 31, and the erosion of rocky coasts 32 .

The versatility of the model allowed us to determine the conditions in which avalanches are powerlaw distributed. Analysis of these cases reveals that the decay (from an initial metastable state) rather consists of successive exponential relaxations, each with a characteristic well-defined size. We demonstrate that a broad distribution of sizes occurs, but that this is not to be associated to long-ranged correlations but to certain randomness. This provides some microscopic support to the old suspicion that observed electronic and magnetic $1 / f$ noises simply consist of a superposition of many different typical scales, each with a different origin. This was argued in Refs. 17, 18, 19, 20]; see also [21] and references therein.

The fact is that our model describes a random combination of event sizes which produces an effective situation which is reminiscent of (equilibrium) critical behavior. Our study does not support, however, a critical condition - neither chaotic behavior - as a source for the many scales in the model. This may have important practical implications.

In spite of its mathematical simplicity, our explanation for the observed many scales is not physically trivial. That is, we describe a time plateau before each avalanche takes place which is to be associated to entropic metastability. On the other hand, we conclude on the existence of dynamic (non-critical) correlations. Furthermore, it ensues that the many scales definitely require randomness in a nonequilibrium setting.

We claim that our findings are consistent with some recent observations, and that it should be possible to test them in purposely designed experiments.

\section{Model and its motivation}

Let the square two-dimensional lattice with binary spin variables at the $N$ sites inside a circle of radius $R$. Interactions are according to the Ising ferromagnetic energy function, $H=-\sum_{\langle i j\rangle} s_{i} s_{j}-h \sum_{i} s_{i}$, where the first sum is over nearest-neighbor pairs of spins. Assume open circular boundary conditions, i.e., bonds leaving the circle are broken. Any configuration $\vec{s} \equiv\left\{s_{i}= \pm 1\right\}$ evolves stochastically with time by spin flips with rate:

$$
\omega\left(s_{i} \rightarrow-s_{i}\right)=p+(1-p) \frac{\mathrm{e}^{-\Delta H_{i} / T}}{1+\mathrm{e}^{-\Delta H_{i} / T}},
$$

where $\Delta H_{i}$ stands for the flip energy cost (we set Boltzmann constant to unity). This amounts to perturb at random, with probability $p$, a canonical tendency to the equilibrium state corresponding to temperature $T$ and energy $H$. This is an efficient, non-trivial way of implementing a complex nonequilibrium situation. That is, dynamics involves a conflict - between finite and "infinite" temperature - which asymptotically drives the system for any $p>0$ towards a nonequilibrium steady state. This essentially differs from the equilibrium situation for $p=033$. Consequently, similar models with competing temperatures have been studied during the last decade as a paradigm of systems far from equilibrium 34 .

The singular behavior that ensues for $p \neq 0$ (in the presence of open boundaries) is, in fact, our main motivation for studying this system. That is, setting $p>0$, even as small as $p \simeq 10^{-6}$, induces a series of successive time plateaus or short-lived "halt states" during the evolution that are not observed for $p=0$. Therefore, (1) in practice provides one of the simplest scenarios one may think of for analyzing in detail a whole class of relaxation phenomena. That is, a principal feature of both this simple model and the cases mentioned in section 1 is that relaxation proceeds by nonequilibrium variations that are realized as jumps between locallystable states.

The present system may be interpreted - though this is not essential to the conclusions below - as an oversimplified model of a small ferromagnetic particle. This, which is relevant to the technology of dense magnetic media 35, 36, 37, requires one to deal with a large surface/volume ratio and, 
consequently, with impurities. In actual specimens, these typically cause perturbations, e.g., diffusion of defects will dynamically disturb the local fields, which one may ideally represent by the random term in (1). In fact, a similar ansatz has already help the understanding of ionic diffusion during magnetic ordering [38], and it has been useful to model microscopic quantum tunneling 39] and non-localized perturbing interactions and fields 33 .

Whichever the specific interpretation is, the model contains a microscopic random perturbation which drives it out of equilibrium. This nonequilibrium randomness, which is likely to characterize also the phenomena of interest (fault slips, electron transport, magnetic domain arrangements,...) happens to induce the most interesting behavior during relaxation. In order to show this, we performed a series of computer simulations. They typically begin with all spins $u p, s_{i}=+1 \forall i$ at $t=0$. For a negative value of the field $h$, this is a metastable state. In fact, for a low enough value of the temperature $T$, the stable state corresponds to $m \equiv N^{-1} \sum_{i} s_{i} \simeq-1$. That is, most of the spins need to flip to point down along the field direction in any stable configuration. For appropriate values of the parameter set $(T, h, p)$ - see below, such a decay consists of a sequence of transitions through short-lived states. This is illustrated in figure 1 for a particle of approximately $10^{3}$ spins at $T=0.25$ (which is about $1 / 10$ the critical temperature of the corresponding infinite system), $h=-0.1$ (1/10 the exchange energy), and a very small value for the perturbation, $p=10^{-6}$.

The same behavior ensues for a broad range of values for $(T, h, p)$. To be more precise, one needs that both domain walls and clusters are well defined. Otherwise, the jumps are difficult to be observed and/or one obtains poor statistics. In order to assure compact configurations and clusters, it turns out necessary a sufficiently low choice for $T$. On the other hand, the parameter $p$ can take a considerable range of values, provided that its effects are comparable to the ones from other stochastic sources. This was observed to occur already for $p=10^{-6}$, which is the value we used in most of our simulation runs (see 40] for other choices). The other model parameter, $h$, just aims at producing metastability, so that only its sign is really relevant. Summing up, the behavior described here is robust

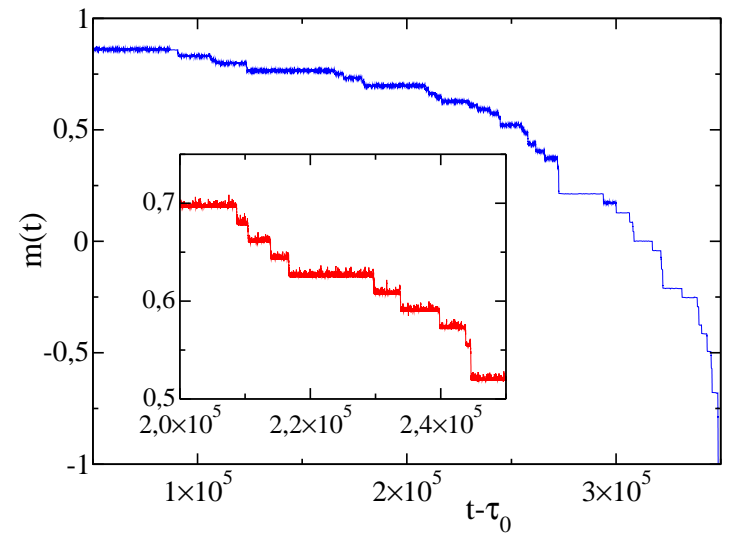

Figure 1: Typical evolution in which the magnetization is observed to decay by jumps to the final stable state. This is for a single particle of radius $R=30$ ( 10 $0^{3}$ spins $)$ at low temperature, and for small values of $h$ and $p$ (see the main text). The time axis shows $t-\tau_{0}$ in MCS (Monte Carlo steps per site) with $\tau_{0}=10^{30} \mathrm{MCS}$; this is of order of the duration of the initial metastable state. The inset shows a significant detail of the relaxation.

within a broad region of parameter space, so that no fine-tuning of parameters is needed.

\section{Some details of relaxation}

Taking the MCS as the relevant, macroscopic time scale, one identifies (e.g., in fig.1) strictly monotonic changes of $m(t)$ that we call "avalanches". Let us define the avalanche duration $\Delta_{t} \equiv\left|t_{a}-t_{b}\right|$ and size $\Delta_{m} \equiv\left|m\left(t_{a}\right)-m\left(t_{b}\right)\right|$, and the associated distributions $P\left(\Delta_{t}\right)$ and $P\left(\Delta_{m}\right)$. We monitored these functions after deducting a trivial noise [11, namely, small thermal events of typical size [40:

$$
\bar{\Delta}=\frac{1}{\ln \left[1+p+(1-p) e^{-2|h| / T}\right]} .
$$

These events correspond to the short-length fluctuations that are evident by direct inspection in the inset of figure 1.

The distribution $P\left(\Delta_{m}\right)$ that results after deducting small events is illustrated in figure 2 . This nicely fits

$$
P\left(\Delta_{m}\right) \sim \Delta_{m}^{-\tau(R)}
$$




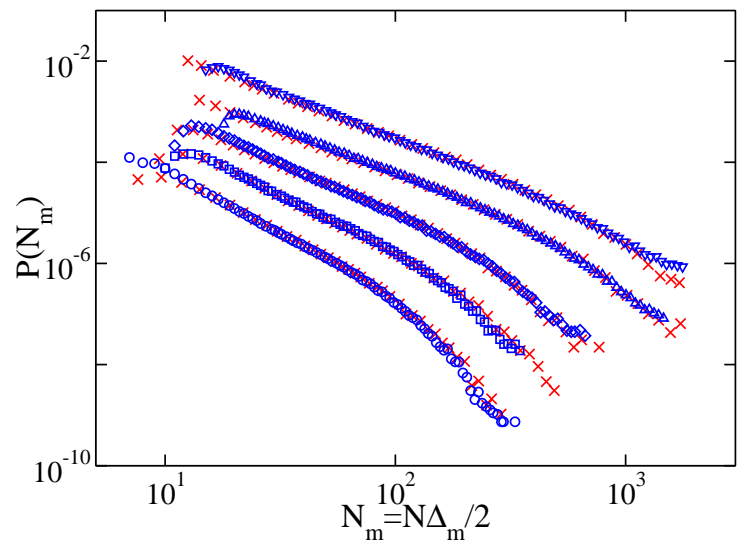

Figure 2: Log-log plot of the size distribution $P\left(\Delta_{m}\right)$ of large avalanches for an ensemble of independent particles of radius (from bottom to top) $R=30,42,60,84$ and 120 , respectively. Plots of the duration distribution $P\left(\Delta_{t}\right)$ vs. $c \Delta_{t}^{\gamma}$ for each $R$ are also shown $(\times)$, with $c \approx 0.5$ and $\gamma \approx 1.52$ (see text). For visual convenience, the curves are shifted vertically by $4^{n}$ with $n=0$ to 4 from bottom to top. Running averages have been performed for clarity purposes.

with

$$
\tau(R)=\tau_{\infty}+a_{1} R^{-2}
$$

where $\tau_{\infty}=1.71(4)$. Figure 3 depicts the corresponding duration distributions. They follow

$$
P\left(\Delta_{t}\right) \sim \Delta_{t}^{-\alpha(R)}
$$

with

$$
\alpha(R)=\alpha_{\infty}+a_{2} R^{-2},
$$

where $\alpha_{\infty}=2.25(3)$. In both cases, size and duration, the apparent power law ends with an exponential tail,

$$
P(\Delta) \sim \exp \left(-\Delta / \bar{\Delta}^{*}\right)
$$

The cutoffs that we observe follow $\bar{\Delta}^{*} \sim R^{\beta}$ with $\beta_{m} \sim 2.32(6)$ and $\beta_{t} \sim 1.53(3)$, respectively (see inset to Fig. 3).

We also determined that observing a power law requires both free borders and the nonequilibrium condition. That is, the distributions $P(\Delta)$ look approximately exponential if the system has periodic borders and/or one sets $p=0$ in eq. (11). This is

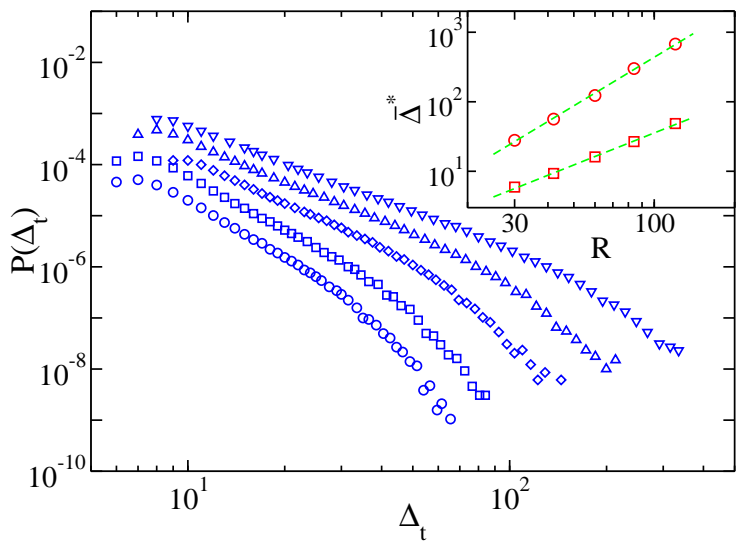

Figure 3: Log-log plot of the duration distribution $P\left(\Delta_{t}\right)$ for the same ensembles of particles as in figure 2. For visual convenience, the curves are shifted vertically by $2^{n}$ with $n=0$ to 4 from bottom to top. Running averages have been performed for clarity purposes. Inset: log-log plot of the size (top) and duration (bottom) cutoffs $\bar{\Delta}^{*}$ vs. $R$. Lines are power-law fits.

discussed below. Another main result is that the observed apparent power laws are here a sum of exponential contributions.

To prove the latter result, we followed the demagnetization process in a large circular particle. The main interest was in the interface between the rich and poor spin-up regions at low temperature. One observes curved interfaces due to the faster growth of the domain near the concave open borders. In fact, the critical droplet always sprouts at the free border [41. Then, given that curvature costs energy, the large avalanches tend to occur at the curved walls, which then transform into rather flat interfaces. We confirmed this by estimating the mean avalanche size $\left\langle\Delta_{m}\right\rangle$ and interface curvature $\langle C\rangle$ as a function of magnetization $m$. The curvature $C$ is defined here as the number of rising steps at the stable-metastable interface 42. We plot in figure 4 our results for these observables. After averaging over many runs, definite correlations show up. That is, as one could perhaps have imagined, the event size is determined by the interface curvature just before the avalanche occurs.

This is confirmed by monitoring $P\left(\Delta_{m} \mid C\right)$, the conditional probability that an avalanche of 


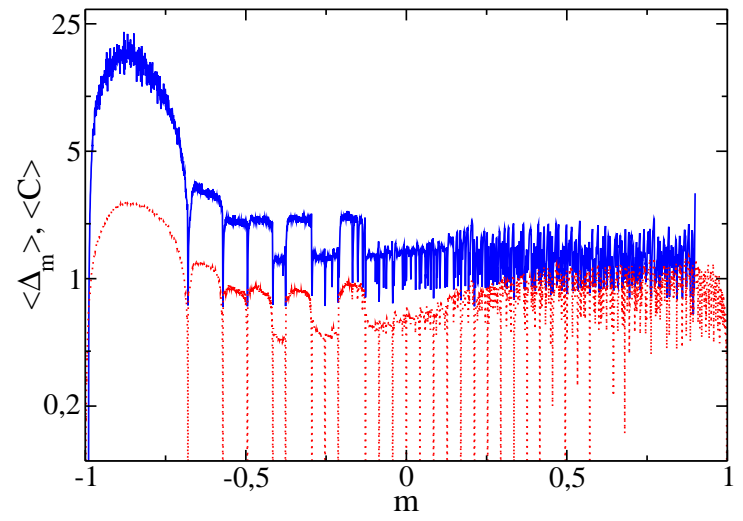

Figure 4: Semilogarithmic plot of $\left\langle\Delta_{m}\right\rangle$ (solid line) and $\langle C\rangle$ (dotted line) as a function of magnetization, after averaging over 3500 independent runs. Notice the non-trivial structure uncovering a high degree of correlation between the mean size of avalanches, $\left\langle\Delta_{m}\right\rangle$, and the average curvature $\langle C\rangle$ of the interface at which the avalanche originates.

size $\Delta_{m}$ develops at an interface region of curvature $C$. We studied this in great detail by simulating an interface of constant curvature evolving by (11). Figure 5 shows that $P\left(\Delta_{m} \mid C\right)$ has two regimes for given $C$. The first one corresponds to the small thermal events mentioned above, namely, those of typical size given by (2). The second regime exhibits, contrary to the situation in figure 2, (stretched-) exponential behavior, namely $P\left(\Delta_{m} \mid C\right) \sim \exp \left[-\left(\Delta_{m} / \bar{\Delta}_{m}\right)^{\eta}\right]$ with $\eta \approx 0.89$. That is, a wall of curvature $C$ induces avalanches of typical size $\bar{\Delta}_{m}(C)$.

This fact turns out most relevant because, due to competition between the randomness induced by free borders and the one induced by $p$ in (1), the interface tends to exhibit a broad range of curvatures with time. More specifically, relaxation proceeds via a series of different configurations, each characterized by a typical curvature of the interface and by the consequent typical form of the critical droplet inducing the avalanche. Therefore, what one really observes when averaging over time is a random combination of many different avalanches, each with its typical well-defined (gap-separated) size and duration, which results in an effective distribution. The fact that this combination depicts several decades (more the larger the system is) of

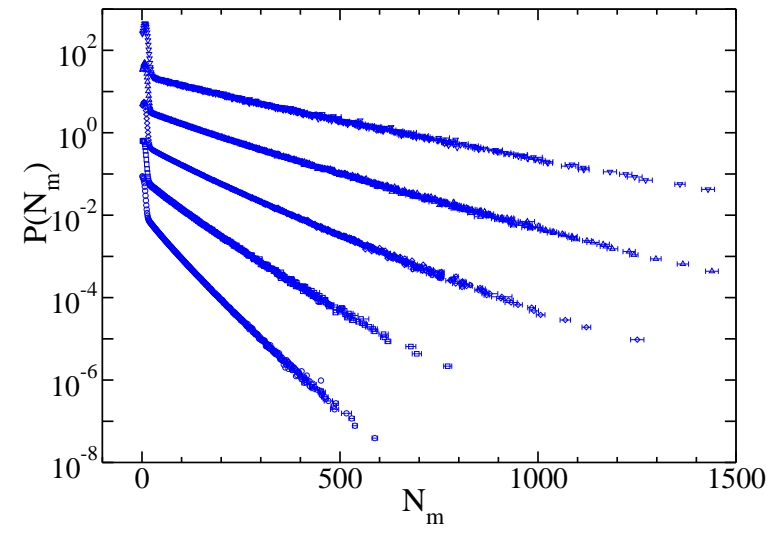

Figure 5: Semilogarithmic plot of $P\left(\Delta_{m} \mid C\right)$, the size distribution for avalanches developing at a wall of constant curvature, $C$; $C$ increases from bottom to top. Here, $N_{m} \equiv \frac{1}{2} N \Delta_{m}$. (For visual convenience, the curves are shifted vertically by $10^{n}$ with $n=0$ to 4 from bottom to top.) Running averages have been performed for clarity purposes.

power-law behavior can be understood on simple grounds.

Let $Q(A)$ the probability of $A$, and $P(x \mid A)=$ $A \exp (-A x)$ the probability of an event of size $x$ given $A$. Assume that $A$ can take a finite number of equally spaced values $A_{k}, k=0,1,2, \ldots, n$, in the interval $\left[A_{\min }, A_{\max }\right]$, so that $A_{k}=A_{\min }+k \delta$ with $\delta=\left(A_{\max }-A_{\min }\right) / n$ (alternatively, one may assume randomly distributed $A_{k} \mathrm{~s}$ ), and that all of them have the same probability, $Q(A)=$ const. One obtains that

$$
\begin{array}{r}
P(x)=\frac{\delta \mathrm{e}^{-x A_{\min }}}{1-\mathrm{e}^{-x \delta}}\left[A_{\min }-A_{\max } \mathrm{e}^{-(n+1) x \delta}\right. \\
\left.-\delta \frac{1-\mathrm{e}^{-n x \delta}}{1-\mathrm{e}^{x \delta}}\right] .
\end{array}
$$

The fact that even such a simple, uncorrelated ansatz describes qualitatively the data is illustrated in figure 6. That is, the superposition of a large but finite number of exponential distributions, each with a typical scale, yields an effective global distribution which is consistent with apparent scale invariance. This distribution extends in practice up to a cutoff, which is also observed in experiments. This cutoff, which corresponds to the slowest exponential relaxation, is given by $\exp \left(-x A_{\min }\right)$. There 
is no evidence that a more involved computation would modify this qualitative conclusions. However, taking into account dynamic correlations as revealed by figure 4 is certainly needed in order to improve quantitative predictions. In particular, eq. (8) predicts a size-independent exponent $\tau(R)=\tau_{\infty}=2$, somewhat different from the observed asymptotic $\tau_{\infty}=1.71(4)$.

Consider now $P\left(\Delta_{t} \mid \Delta_{m}\right)$, i.e., the probability that the avalanche of size $\Delta_{m}$ lasts a time $\Delta_{t}$. We confirmed that this exhibits well-defined peaks corresponding to large correlations, i.e., avalanches of a given size have a preferred duration and vice versa. Assuming $\Delta_{m} \sim \Delta_{t}^{\gamma}$, we obtain $\gamma=$ $\beta_{m} / \beta_{t}=1.52(5)$. Using this relation, one may obtain the duration distribution by combining (8) with $P\left(\Delta_{m}\right) \mathrm{d} \Delta_{m}=P\left(\Delta_{t}\right) \mathrm{d} \Delta_{t}$. A comparison of the resulting curve with data in Fig. [6 leads to $\gamma \simeq 1.52$, in agreement with the value obtained from the cutoff exponents $\beta$. More generally, a scaling plot of $P\left(\Delta_{t}\right)$ vs. $c \Delta_{t}^{\gamma}$, with $c$ some proportionality constant, must collapse onto the corresponding curve $P\left(\Delta_{m}\right)$ for each $R$. This is confirmed in Fig. 2 for $\gamma \simeq 1.52$, further supporting the scale-superposition scenario. On the hypothesis that both $P\left(\Delta_{m}\right)$ and $P\left(\Delta_{t}\right)$ were true power-law distributions, one would obtain the scaling relation $(\alpha-1)=\gamma(\tau-1)$. However, our values above for $\alpha$ and $\tau$ would imply here that $\gamma \simeq 1.76$. This misfit is a consequence of the fact that, according to our point in this paper, none of the distributions $P(\Delta)$ exhibits true scaling behavior.

\section{Discussion}

The model studied in this paper has been demonstrated to relax only via well-defined nearexponential events. Each has its own scale, but many of them randomly combine into a distribution that exhibits a power-law portion. This occurs in the model because two of its features, namely, $p \neq 0$ and free borders. Otherwise, i.e., $p=0$ and/or periodic boundary conditions, the apparent power law does not emerge, but one observes a well-defined mean. Therefore, the cause for a relatively broad range of possible different scales is the underlying nonequilibrium randomness that characterizes the model. The question is whether this picture applies also to reported scale-free fluctua-

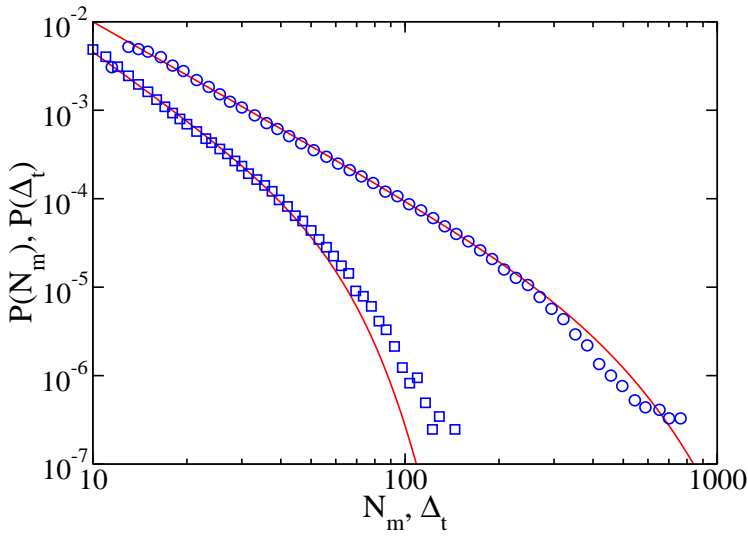

Figure 6: Solid lines are predictions from equation (8) for $n=200, A_{\min }=0.007$ and $A_{\max }=1$. The symbols stand for the avalanches duration (lower curve) and size (upper curve) when $R=60$, i.e., two of the data sets in figures 2 and 3 . In this particular case, the finite-size exponent is $\tau(R=$ $60)=2.06(2)$, allowing direct comparison with eq. (8).

tions in many natural phenomena, where it is difficult to investigate separate elementary events. We show below that there are some indications - but not a proof - that this may be the case. We also remark in this section some important features of our picture that one should look for in experiments.

We first remark that analyzing the fluctuations of interest requires a previous scrutiny of data in the model separating small from larger events. The former are random events of well-defined size according to eq. (2). The latter are more structured, correlated events or "avalanches". This separation is theoretically motivated [2, 11], and it is also supported by experiments [28, 43]. The avalanches are then accurately described by an apparent power law. This involves an exponent and a cutoff both depending on the size of the system. Interesting enough, there is a well-defined limit for a macroscopic particle. That is, even though free borders (a surface effect) are essential to the phenomena, increasing the particle to macroscopic will not prevent one from observing all scales, including very large, say macroscopic avalanches.

Failing in separating accurately small from large events will, in general, result in a non-significative 
distribution. On the other hand, though the described behavior is robust within a broad region of parameter space, unambiguously observing the relevant phenomena requires some care in order to have compact enough clusters and well-defined interfaces. As indicated in section 1] prevalence of one ingredient over the others may importantly hamper statistics which may obscure the situation. These two facts - together with other agents 44 (see below) - seem to produce both power-law and exponential distributions in closely related situations as described, for instance, in [2].

There is no indication that the apparent scale invariance that we observe is to be associated with chaos, e.g., sensitivity to the initial condition. Our picture is neither consistent with a critical condition. Criticality implies a diverging correlation length which is not detected in our simulations. Instead, the many scales in the model are simply due to the underlying nonequilibrium randomness mentioned above. A balance between random and nonequilibrium features has already been claimed to be essential for SOC in different settings [2, 15, 44, 45, 46, 47. Our study suggests this is the essential physics in a family of situations. That is, even though details may vary, e.g., from random interface rearrangements in magnets to slip complexity in earthquakes, there is always some microscopic randomness which induces multiple short-lived situations. This constantly halts the decay and, ultimately, leads to apparent scale invariance.

We find it remarkable - even though it does not prove anything - that the statistical properties of the avalanches in our model are indistinguishable in practice from what has been reported for some detailed laboratory experiments. For instance, size corrections similar to the ones in (4) and (6) for $\tau$ and $\alpha$, respectively, have been reported in avalanche experiments on rice piles [4]. Moreover, our values for the infinite case are strikingly close to the ones reported in magnetic experiments, e.g., $\tau_{\infty}=1.77(9), \alpha_{\infty}=2.22(8)$ and $\gamma=1.51(1)$ in Ref. [1] for quasi-two dimensional systems. (See also Ref. 43.) On the other hand, our cutoff values in (7) follow the precise trend observed in magnetic materials [4, 49]. More qualitatively, one may argue that the available literature on the Barkhausen noise, which is rather proclive to a hypothetical critical point, provides meaningful indications of consistency with our non-critical behavior, as reported elsewhere 40. If this is granted, the many transitions through halt states in our picture would correspond to topological rearrangements of domain walls in the Barkhausen case. In fact, there is also in this case a domain finite size and a nonequilibrium perturbing drive (the varying field), that would induce a condition similar to our "nonequilibrium randomness" constantly changing the scale.

Avalanches in our model do not continue indefinitely in time but disappear when the system reaches the stable state. This contrasts in principle with the stationary character of some experimental signals. However, it does not prevent our comparison. To illustrate this, imagine that we flip the magnetic field sign in our model every time the system reaches the final stable state. This would give rise to a cyclic steady process, much in the spirit of hysteresis experiments in magnets. Avalanches observed in this cyclic state have the same properties that the ones reported here. In particular, our conclusions on the origin of the apparent scaling of distributions remain unchanged, while the process is now cyclically stationary.

No doubt it would be interesting to study the possible occurrence of "short-lived halt states" in nature. These are associated in the model with flat interfaces. That is, once the initial metastability breaks down, the particle becomes inhomogeneous, and flat interfaces have a significant probability to form after each avalanche (which aims at minimizing interfacial energy). As this is the most stable configuration against small perturbations, the system remains some time with constant magnetization $m\left(t_{b}\right)$. This may be described as an entropic metastability. There is no real energy barrier but an unstable situation such that a given microscopic random event suffices to initiate the next avalanche.

Interesting enough, this picture gives more hope to the goal of predicting large events. That is, we claim that catastrophes are not a rare random emergence in an strongly correlated bulk which, consequently, have the same cause as the small events. 1, 2, 3, 4] Instead, events are characterized by their size, and each size follows from some specific microscopic configuration. The configurations that, under appropriate conditions, may originate large events qualitatively differ from the ones corresponding to smaller events. In summary, there is 
some specific cause for each event which depends on its size. Consider for instance a stock market; its evolution is also characterized by discontinuous, sudden jumps between different locally-stable states. In order to predict a crash in this system, assuming that our picture applies, one should look for the simultaneous occurrence of a large flat interface (predisposition of the players) and macroscopic free borders (some large external perturbation). Studying the statistical properties of the many events will then only inform on the relative probability of each microscopic relevant configuration.

A detailed description of the scape mechanism from entropic metastability could relate our picture to other approaches. We mention in this respect that, after averaging over many independent particles, the lifetimes of the halt states during the relaxation of our model depict an exponential distribution. Therefore, they show a typical scale. This scale turns out to be much shorter than the time scale for the system relaxation, as reported to characterize the supposed nonequilibrium criticality which is assumed to underly many $1 / f$ noises; see, for instance, Ref. 2]. An even more detailed look, which requires averaging over time intervals, reveals in our model that this scale definitely decreases with $t$ from, say, macroscopic ( $\gtrsim 10^{5}$ MCS) to microscopic ( $10 \mathrm{MCS})$. This feature, which is already evidenced by (direct inspection of) figure 1 , is one that could perhaps be easily detected in experiments.

Finally, we remark that there are other possible explanations for $1 / f$ noise based on non-critical mechanisms; see, for example, Refs. 21]-[26]. These are less general than the mechanism proposed here, and often restricted to some very specific situation. Furthermore, some of these descriptions may be interpreted at the light of a superposition of many different typical scales, as in our mechanism. A similar origin for electronic $1 / f$ noises was suggested in the past (see, for instance, Refs. [17, 18, 19, 20, 21]), though the present paper is, to our knowledge, the first one in establishing an explicit relation between elementary events (avalanches) and microscopic physical processes.

We acknowledge M.A. Muñoz for very useful comments, and financial support from MEyC, project FIS2005-00791.

† Present address Physics Department, Boston University, Boston, MA 02215, USA

\section{References}

[1] P. Bak, How Nature Works, Copernicus, N.Y. 1996.

[2] H.J. Jensen, Self-Organized Criticality, Cambridge Univ. Press, Cambridge 1998.

[3] R.N. Mantegna and H.E. Stanley, An Introduction to Econophysics: Correlations and Complexity in Finance, Cambridge Univ. Press, Cambridge 2000.

[4] D. Sornette, Critical Phenomena in Natural Sciences, Springer-Verlag, Heidelberg 2000.

[5] G.B. West and J.H. Brown, Phys. Today 57, $36(2004)$

[6] W. Cook, P. Ormerod and E. Cooper, J. Stat. Mech.: Theor.Exp. P07003 (2004)

[7] A.E. Motter, Phys. Rev. Lett. 93, 098701 (2004)

[8] J.B. Johnson, Phys. Rev. 26, 71 (1925)

[9] W. Schottky, Phys. Rev. 28, 74 (1926)

[10] H. Barkhausen, Z. Phys. 20, 401 (1919)

[11] D. Spasojevic, S. Burkvic, S. Milosevic, and H.E. Stanley, Phys. Rev. E 54, 2531 (1996)

[12] S.A. Kauffman, The Origins of Order, Oxford Univ. Press, N.Y. 1993.

[13] O. Perkovic, K. Dahmen and J. Sethna, Phys. Rev. Lett. 75,4528 (1995)

[14] P. Cizeau, S. Zapperi, G. Durin, and H.E. Stanley, Phys. Rev. Lett. 79, 4669 (1997)

[15] A. Vespignani, R. Dickman, and M.A. Muñoz, Phys. Rev. Lett. 81, 5676 (1998)

[16] J.M. Carlson and J. Doyle, Phys. Rev. Lett. 84, 2529 (2000); M. Newman, Nature 405, 412 (2000)

[17] J. Bernamont, Ann. Phys. (Leipzig) 7, 71 (1937)

[18] H. Sawada, J. Phys. Soc. Japan 7, 575 (1952)

[19] P. Mazzetti, Il Nuovo Cim. 25, 1322 (1962); ibid 31, 88 (1964) 
[20] I. Procaccia and H. Schuster, Phys. Rev. A 28, R1210 (1983)

[21] M.B. Weissman, Rev. Mod. Phys. 60, 537 (1988)

[22] A. Bunde et al, Phys. Rev. Lett. 78, 3338 (1997)

[23] P. De Los Rios and Y.-C. Zhang, Phys. Rev. Lett. 82, 472 (1999)

[24] B. Kaulakys, Microelectr. Reliab. 40, 1787 (2000); also as ArXiv cond-mat/0305067

[25] J. Davidsen and H.G. Schuster Phys. Rev. E 62, 6111 (2000); ibid 65, 26120 (2002)

[26] E. Milotti, Phys. Rev. E 51, 3087 (1995)

[27] J. Laherrère and D. Sornette, Eur. Phys. J. B 2, 525 (1998)

[28] M. De Menech, A.L. Stella, and C. Tebaldi, Phys. Rev. E 58, R2677 (1998); C. Tebaldi, M. De Menech, and A.L. Stella, Phys. Rev. Lett. 83, 3952 (1999); M. De Menech and A.L. Stella, Phys. Rev. E 62, R4528 (2000)

[29] X. Che and H. Suhl, Phys. Rev. Lett. 64, 1670 (1990)

[30] K.L. Babcock and R.W. Westervelt, Phys. Rev. Lett. 64, 2168 (1990)

[31] P. Bak, K. Christensen, L. Danon, and T. Scanlon, Phys. Rev. Lett. 88, 178501 (2002); M.S. Mega, P. Allegrini, P. Grigolini, V. Latora, L. Palatella, A. Rapisarda, and S. Vinciguerra, Phys. Rev. Lett. 90, 188501 (2003)

[32] B. Sapoval, A. Baldassarri,and A. Gabrielli, Phys. Rev. Lett. 93, 098501 (2004)

[33] J. Marro and R. Dickman, Nonequilibrium Phase Transitions, Cambridge Univ. Press, Cambridge 1999.

[34] See, for instance, P.L. Garrido, A. Labarta and J. Marro, Journal of Statistical Physics, 49 551-568 (1987); K.E. Bassler and Z. Rácz, Phys. Rev. Lett. 73, 1320 (1994); P. Tamayo, F.J. Alexander, and R. Gupta Phys. Rev. E 50, 3474 (1994); A. Szolnoki, G. Szabó, and O.G. Mouritsen, Phys. Rev. E 55, 2255
(1997); B. Schmittmann and F. Schmüser, Phys. Rev. E 66, 046130 (2002); P. I. Hurtado, P. L. Garrido, and J. Marro, Phys. Rev. B, 70, 245409 (2004); P.I. Hurtado, J. Marro, and P.L. Garrido, Phys. Rev. E 70, 021101 (2004).

[35] K.S. Novoselov, A.K. Geim, S.V. Dubonos, E.W. Hill, and I.V. Grigorieva, Nature 426, $812(2003)$

[36] J.L. Simonds, Phys. Today 48, 26 (1995)

[37] J. Shi, S. Gider, K. Babcock, and D. Awschalom, Science 271, 937 (1996)

[38] P.L. Garrido, J. Marro, and J.J. Torres, Phys. Rev. B 58, 11488 (1998)

[39] J. Marro and J.A. Vacas, Phys. Rev. B 56, 8863 (1997)

[40] P.I. Hurtado et al., to be published.

[41] E. Cirillo and J.L. Lebowitz, J. Stat. Phys. 90, 211 (1998)

[42] That is, the number of up spins flanked, respectively, by two ups and by two downs at the sides along the interface. This definition requires well-defined compact clusters, as for low temperature.

[43] G.-P. Zheng, M. Li, and J. Zhang, J. Appl. Phys. 92, 883 (2002)

[44] V. Frette, K. Christensen, A. MaltheSorenssen, J. Feder, T. Jossand, and P. Meakin, Nature 379, 49 (1996)

[45] D. Dhar, Physica A 264, 1 (1999)

[46] R. Dickman et al, J. Phys. 30, 27 (2000); Physica A 306, 90 (2002)

[47] M.Alava, J. Phys.: Cond.Matt. 142353 (2002)

[48] M.Bahiana et al., Phys.Rev. E59, 3884 (1999)

[49] G. Durin and S. Zapperi, Phys. Rev. Lett. 84, 4705 (2000) 